\title{
FIELD TRIALS AND TEST RESULTS OF PORTABLE DVB-T SYSTEMS WITH TRANSMIT DELAY DIVERSITY
}

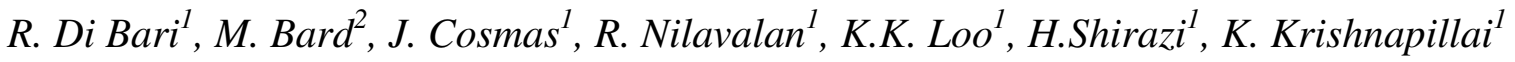 \\ 1) School of Engineering and Design, Brunel University \\ Uxbridge, Middlesex, (UK) \\ 2) Broadreach Systems, Torquay, (UK)
}

\begin{abstract}
This paper describes work carried out by Brunel University and Broadreach Systems (UK) to quantify the advantages that can be achieved if Transmit Diversity is applied to systems employing the DVB standard. The techniques investigated can be applied to standard receiver equipment without modification. An extensive and carefully planned field trial was performed during the winter of $2007 / 2008$ in Uxbridge (UK) to validate predictions from theoretical modeling and laboratory simulations. The transmissions were performed in the $730 \mathrm{MHz}$ frequency band with a DVB-T transmitter and a mean power of $18.4 \mathrm{dBW}$. Transmit delay diversity has been observed to deliver significant reception improvement in automotive and indoor- non line of sight situations.
\end{abstract}

Index Terms - Field trials, Transmit Delay Diversity, DVB-T

\section{INTRODUCTION}

Multipath can result in severe fading in Non Line of Sight (NLOS) reception situations. A radio network design must take this into account when predicting service availability. Traditionally broadcast reception in multipath situations is provided by increasing the density of transmitters and increasing the radiated power. Transmit diversity is a technique where multiple transmitters, separated by only a few meters deliver signals to a NLOS receiver along radio channels with uncorrelated fading. The effect is to reduce the probability of observing deep fades at the receiver. A diversity gain will always be observed in NLOS situations provided the observed signals have uncorrelated fading and the receiver is able to exploit them, the result is that the number of transmitter sites and radiated power required to achieve effective coverage are both reduced. In this paper, an extensive and carefully planned field trial performed during the winter of $2007 / 2008$ in Uxbridge (UK) is described. The transmissions were performed in the $730 \mathrm{MHz}$ frequency band with a DVB-T transmitter and a mean power of $18.4 \mathrm{dBW}$. These trials were organized by Brunel University and Broadreach Systems.

\section{EXPERIMENTAL NETWORK}

The transmitter was located at Uxbridge's Brunel University, in the west outskirts of London, 1.2 Km south of Uxbridge. The measurement area is relatively flat and representative of a typical sub-urban area. Our field trial used two power amplifiers, rated at $100 \mathrm{~W}$, feeding an array of directive antennas.

In the tests we compared quality of reception in the measurement area when either all power was transmitted from a single antenna (Single In Single Out) or half power from each of 2 separate antennas (Multiple In Multiple Out). The effective isotropic radiated power (EIRP) was $18.4 \mathrm{dBW}$ for both the single transmitter and dual transmitter (MISO) configurations on channel 53 which has a central frequency of $730 \mathrm{MHz}$ and bandwidth of 8 MHz. The signals were compliant with the RF signal spectrum of DVB-T standard [2]. A directive antenna linear array was installed at the top of one of the university buildings (Tower D) pointing toward the east The antennas are horizontally polarized panels with a gain of $12 \mathrm{~dB}$, a half-power beam-width of $53^{\circ}$ degrees in the $\mathrm{H}$ plane and $24^{\circ}$ degree in the $\mathrm{V}$ plane. A maximum number of two antennas (out of five) were active at any time. The spatial separations between active antennas could be selected from 8 to 27 wavelengths (Fig. 1).

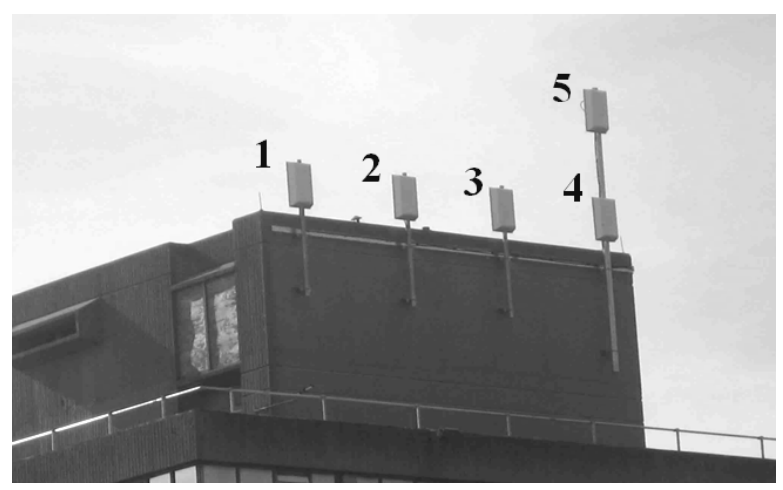

Fig. 1. Brunel's Diversity Antenna Array

The total feeder loss was $6.8 \mathrm{~dB}$ and the switch matrix connecting the amplifier B with one of the diversity antennas had a loss of $0.2 \mathrm{~dB}$. In Table 1 the main technical characteristics of the transmission are summarized.

Pseudo Random Binary Sequence (PRBS) packets were used for field measurements. The system is also capable of delivering multimedia content, encoded into MPEG-2 Transport Streams 


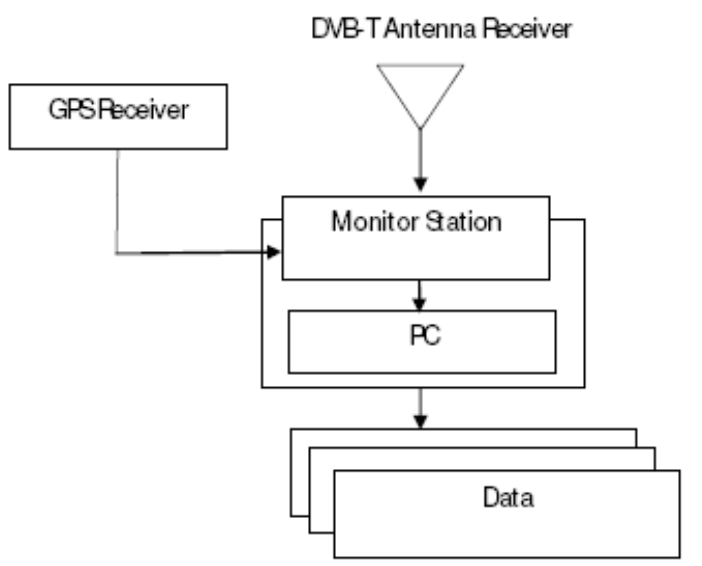

Fig. 2. The car experimental set-up

(TS) packets and transmitting it over the network for demonstration purposes. A standard DVB-T Modulator was used to feed IF signals directly into the Broadreach Diversity Unit (custom made), introducing a $1.1 \mu$ s relative delay between the two signals at the output.

The mobile reception was measured using a car equipped with a DVB measurement system provided by Broadreach Systems. Fig. 2 illustrates the integrated system used to record seconds in which uncorrectable Reed-Solomon blocks are observed, from this the Errored Seconds Ratio (ESR) is calculated, this is the ratio of errored to non-errored seconds. The Signal to Noise Ratio (SNR) and the received signal strength indication (RSSI) are also measured together with location coordinates acquired using a global position system (GPS) receiver. The Broadreach monitor receiver comprised a DVB-T demodulator measuring the signal quality, an integral processor to store data and provide user interfaces, and a GPS receiver. The signal was received using an omnidirectional horizontally polarized antenna, installed on the rooftop of the test vehicle. This system was used to measure the ESR, SNR, and RSSI, every measurement was recorded and tagged with the corresponding location coordinates.

\section{MEASUREMENT METHODOLOGY}

\subsection{Measurement metrics}

A complete set of radio channel and signal data were gathered every second. The parameters of interest include the Errored Seconds (ES), the RSSI, the SNR and the Channel Impulse Response (CIR). The ES denotes the number of seconds containing faulty MPEG-TS packets. Errored seconds are a good metric as they are observable in the received picture and therefore reflect the impact of signal quality on the subscriber's experience. A total errored second ratio (ESRT) per each experiment is defined as:

$$
\operatorname{ESRT}[\%]=\frac{E S}{\text { total number of measurement sec onds }} \times 100 .
$$

The ESRT gain relative to SISO is defined as

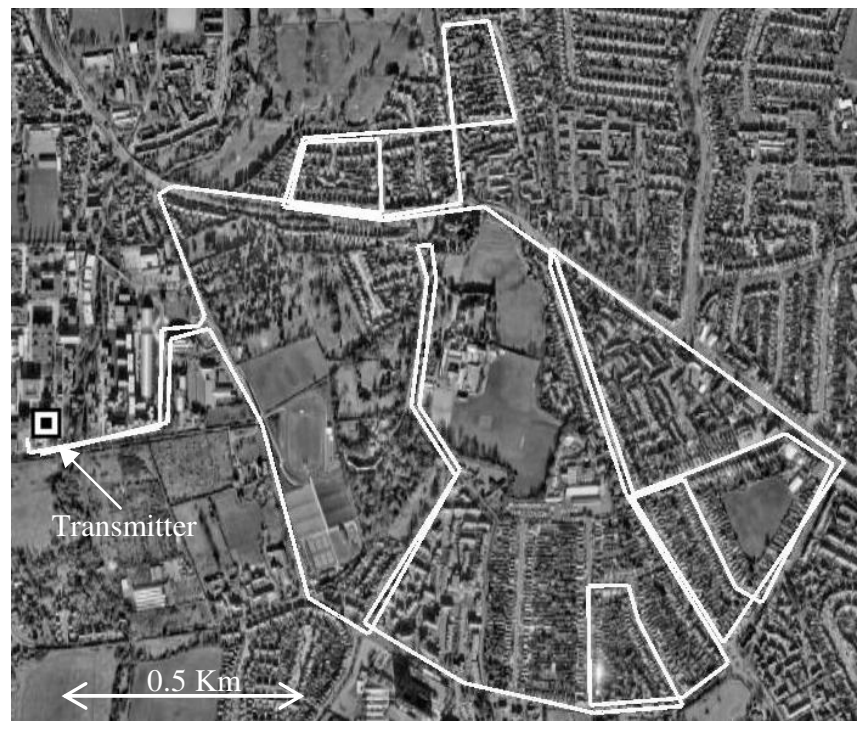

Fig. 3. The selected route and transmit antenna's 3dB beamwidth.

$$
G_{E S R T, S I S O}[\%]=\frac{G A I N_{E S R T}}{E S R T_{\text {SISO }}} \times 100,
$$

where $G_{E S R T}[\%]=E S R T_{\text {SISO }}-E S R T_{M I S O}$. Reference [3] defines the ESR5 metric (erroneous seconds ratio at 5\%) to denote a maximum of one erroneous second in 20 seconds as the maximum rate for acceptable reception. Thus the percentage of good reception $R$ during each experiment is defined as

$$
R[\%]=\frac{\text { total number of ESR5 clusters }}{\text { total number of } 20 \text { seconds clusters }} \times 100 .
$$

The reception gain $G_{R}$ is defined as

$$
G_{R, S I S O}[\%]=\frac{G_{R}}{R_{\text {SISO }}} \times 100,
$$

where $G_{R}[\%]=R_{\text {SISO }}-R_{\text {MISO }}$.

The channel impulse response (CIR) is measured at the beginning of each second and comprises 77 SNR measurements in the time delay domain with a resolution of $74 \mathrm{~ns}$.

\subsection{Area classification and route description}

The selected route covered mainly sub-urban zones and included wide and medium roads in all directions reaching marginal areas of Brunel's service coverage. The route was in the main direction of the transmit power, it is approximately $13.2 \mathrm{Km}$ long and was selected mainly to measure the diversity gain. The maximum range covered by the route was $1.72 \mathrm{Km}$. It should be mentioned that the measurements were repeated many times on the same routes to confirm repeatability. In total more than $447 \mathrm{~km}$ of mobile measurements were recorded. Fig. 3 shows the selected route. Typical speeds were in the range of 0 to $48.2 \mathrm{Km} / \mathrm{h}$, with an average of $30 \mathrm{Km} / \mathrm{h}$. The measurements taken when the receiver was stationary were removed from the data presented here to eliminate the effects of random stops due to traffic lights etc on the total reception statistics. 


\subsection{Verification of the transmitter set-up}

The signal quality was tested at several stages in the transmit chain to ensure it was acceptable. The relative levels of the spectrum shoulders are fully compliant with the RF signal spectrum mask of DVB-T standard [2]. The two amplifiers did not introduce any significant non-linear effects when operating on SISO configuration (e.g. at full power). The power transfer system was calibrated and the power levels were carefully measured, adjusted and monitored during the measurements using a power measurement device with calibration traceable to national standards. In the MISO case each antenna transmitted half of the EIRP of the SISO case.

\section{MOBILE RECEPTION RESULTS}

The time required for driving along the route was approximately 30 minutes. The diversity gain was estimated by comparing SISO and MISO driving tests performed sequentially to ensure analogous traffic conditions for the two measurements. Weather conditions were sunny or partly cloudy and background traffic was generally light/medium. The tests results presented here were obtained employing the broadcast conditions described in Table 1.

Table 1. DVB-T system parameters

\begin{tabular}{|l|l|l|}
\hline Configuration Name & SISO & MISO \\
\hline Code Rate & $3 / 4$ \\
\hline Signal Constellation & 4-QAM \\
\hline DVB-T Mode & $2 \mathrm{k}$ \\
\hline Hierarchy $(\alpha)$ & Non hierarchical \\
\hline Guard Interval & $1 / 32$ & \multicolumn{2}{|l|}{} \\
\hline Bitrate $($ Mbit/s) & 9.05 & $\begin{array}{l}15.4(\text { ant. } 1)+15.4 \\
\text { (ant. 4) }\end{array}$ \\
\hline EIRP $(\mathrm{dBW})$ & 18.4 & 24 \\
\hline Antenna separation $(\lambda)$ & - & 1.1 \\
\hline Diversity Delay $(\mu s)$ & - & \\
\hline
\end{tabular}

\subsection{Performance of Transmit Diversity}

Fig. 4 shows the percentage of good Reception (where ESR is equal or less than 5\%) for SISO and MISO (left axis) and their relative $G_{E S R T}$ and $G_{E S R T \text { SISO }}$ (right axis). The measurements were taken during different days, and arranged with $G_{R}$ descending. A $90 \%$ confidence interval is derived from Student's t-distribution. The sample mean $G_{R}$ is about $14.3 \%$ with a sample variance of $7.4 \%$ and a $90 \%$ confidence interval lower and upper bound equal to $4.8 \%$ and $23.8 \%$, respectively. The sample mean $G_{R, S I S O}$ is about $22.2 \%$ with a sample variation of $12.4 \%$ and a $90 \%$ confidence interval lower and upper bound equal to $9.9 \%$ and $34.4 \%$, respectively. In Table 2 the means, variances and $90 \%$ confidence interval are summarized.

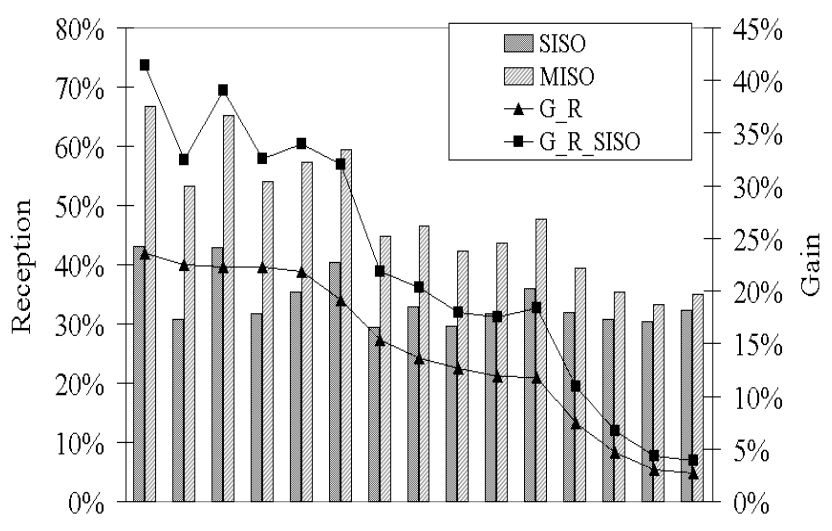

Fig. 4. Reception of all experiments (left axis) with $G_{R, S I S O}$ and $G_{R}$ (right axis).

As described in the previous section, $G_{E S R T, S I S O}$ and $G_{R, S I S O}$ consist of the $G_{E S R T, S I S O}$ and $G_{R, S I S O}$ normalized by the SISO performance (e.g. $E S R T_{\text {SISO }}$ and $R_{S I S O}$ ). The data high variances are due to different the traffic conditions for the measurements compared. However, the results clearly show that the MISO performances were always better than SISO.

Table 2. Sample mean and variance of the gain measurements and confidence intervals derived from Student's t-distribution

\begin{tabular}{|c|c|c|c|c|}
\hline & \multirow{2}{*}{$\begin{array}{l}\text { sample } \\
\text { mean } \\
{[\%]}\end{array}$} & \multirow{2}{*}{$\begin{array}{l}\text { sample } \\
\text { variance } \\
{[\%]}\end{array}$} & \multicolumn{2}{|l|}{$\begin{array}{l}90 \% \\
\text { intervals }\end{array}$} \\
\hline & & & $\begin{array}{l}\text { lower } \\
\text { bound [\%] }\end{array}$ & $\begin{array}{l}\text { upper } \\
\text { bound } \\
{[\%]}\end{array}$ \\
\hline$G_{E S R T}$ & 14.9 & 4.9 & 7.2 & 22.6 \\
\hline$G_{E S R T, S I S O}$ & 31.1 & 11.3 & 19.4 & 42.8 \\
\hline$G_{R}$ & 14.3 & 7.4 & 4.8 & 23.8 \\
\hline$G_{R, S I S O}$ & 22.2 & 12.4 & 9.9 & 34.4 \\
\hline
\end{tabular}

Fig. 5 shows how transmit power diversity gain has been estimated. The ESR for SISO transmission was measured first. The total ERP for MISO transmission was then reduced in $1 \mathrm{~dB}$ steps until the observed ESR was equivalent to the SISO case. The transmit power gain is the reduction in MISO ERP required to achieve MISO ESR equivalent to the SISO case. The test was performed on three separate days, Fig. 8 shows the SISO ESR measured on each day and also the MISO ESR at each of the ERP levels. The gain relative to SISO has been estimated by a linear regression forecast of the MISO reception. The results show a gain of $3.4 \mathrm{~dB}, 3.9 \mathrm{~dB}$, and $4.0 \mathrm{~dB}$. Table 3 shows the SISO root mean square delay spread (DS) calculated for the COST 207 TU6 and RA channel models and compares this to the rms DS measured during our driving tests. It can be seen that the measured DS is $0.24 \mu s$ and is between the TU6 and RA figures. The second part of Table 3 compares the diversity gain measured in a simulated environment for TU6 and RA at a Doppler Frequency of $1 \mathrm{~Hz}$, compared to the gain measured in our driving tests. 


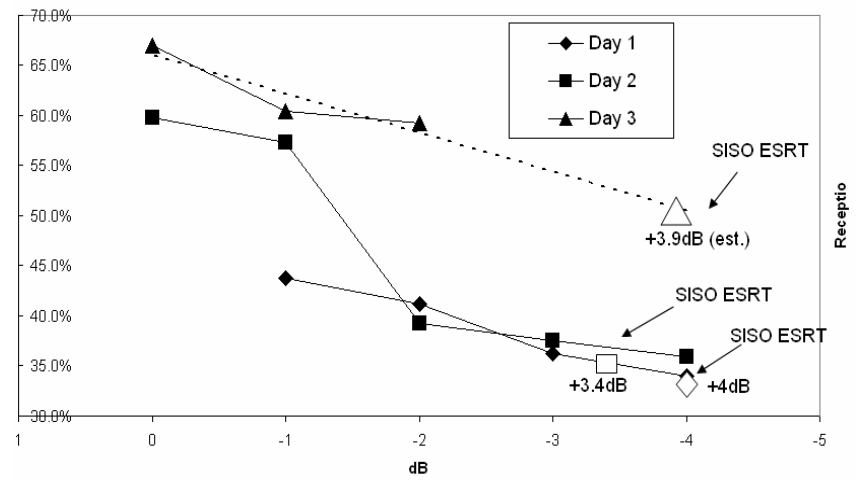

Fig. 5. Transmit power gain

It can be seen that the estimated diversity gain is within the expected range of $2-5.2 \mathrm{~dB}$, thus validating our measurements and processes.

Table 3. Delay Spread and transmit power gain

\begin{tabular}{|c|c|c|c|c|c|}
\hline \multicolumn{3}{|c|}{$\begin{array}{c}\text { SISO DS } \\
(\mu s)\end{array}$} & \multicolumn{3}{|c|}{$\begin{array}{c}\text { Gain at ESR5 } \\
(\mathrm{dB})\end{array}$} \\
\hline \multicolumn{2}{|c|}{ COST 207} & \multirow{2}{*}{ Estimated } & \multicolumn{2}{|c|}{ COST 207} & \multirow{2}{*}{ Estimated } \\
\hline TU6 & $\mathrm{RA}$ & & $\begin{array}{r}\text { TU6, } \\
F_{D}=1 \mathrm{~Hz} \\
\end{array}$ & $\begin{array}{c}\mathrm{RA}, \\
\mathrm{F}_{\mathrm{D}}=1 \mathrm{~Hz}\end{array}$ & \\
\hline 1 & 0.1 & 0.24 & 2.0 & 5.2 & $\begin{array}{c}3.4,3.9 \\
\text { and } 4\end{array}$ \\
\hline
\end{tabular}

Measurements have also been conducted to explore how Tx Diversity might be used to enhance system throughput capability. SISO and MISO performance was compared at 3 different code rates of $1 / 2,2 / 3$ and $3 / 4$, the tests were repeated on 3 separate days. Fig. 6 shows the percentage of good reception (R) for each of the tests and shows that SISO reception at a code rate of $1 / 2$ is roughly comparable to MISO reception at a code rate of $2 / 3$ or better. Since code rates of $1 / 2,2 / 3$ and $3 / 4$ have corresponding bitrates of $6.03,8.04$, and $9.05 \mathrm{Mbit} / \mathrm{s}$, the measurements suggest that an average improvement in data throughput of at least 2.Mbit/s or $30 \%$ can be achieved.

\section{CONCLUSIONS}

The results presented here show that transmit diversity is effective when applied to a standard DVB-T system and that performance predicted by theoretical modeling and laboratory simulations can be realized in real World situations. The results show that, when driving in a sub-urban area, the number of errored seconds observed is greatly reduced and instances of acceptable reception are significantly increased. The diversity gain in terms of ERP has been measured and found to agree with theoretical predictions. Finally, it has been shown that transmit Diversity can be used to provide a data throughput improvement of $30 \%$ or more.

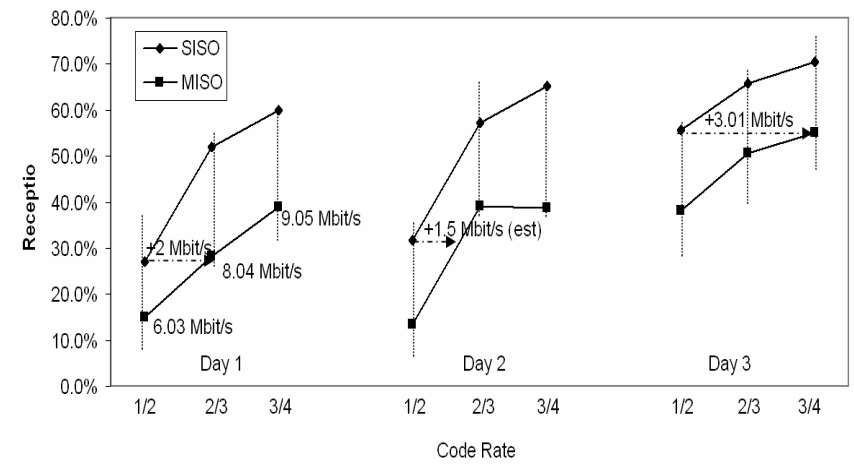

Fig. 6. SISO bitrates compared with MISO for different code rates

Future measurement campaigns will explore the effectiveness of Tx diversity for indoors reception compared to theoretical predictions and evaluate the impact on radio network design.

The tests and results presented here are for techniques that can be applied to Broadcasting systems utilising existing DVB standards without modification, the advantages can be exploited at the network level since there is no impact on receiver design. Resulting DVB broadcast networks could have fewer transmitter sites, lower EIRP or support higher data throughput due to the increased and thus would be more cost-effective and have less environmental impact.

\section{REFERENCES}

[1] Y. Zhang, J. Cosmas, K.-K. Loo, M. Bard, and R. D. Bari, "Analysis of Cyclic Delay Diversity on DVB-H Systems over Spatially Correlated Channel," IEEE Transactions on Broadcasting, Vol.53, No.1, March 2007.

[2] R. Di Bari, M. Bard, Y. Zhang, K.M. Nasr, J. Cosmas, K.K. Loo, R. Nilavalan, H. Shirazi, K. Krishnapillai, "Laboratory Measurement Campaign of DVB-T Signal with Transmit Delay Diversity," to appear in IEEE Transactions on Broadcasting Special Issue on "Quality Issue in Multimedia Broadcasting", 2008.

[3] European Telecommunications Standard Institute ETSI, Digital Video Broadcasting (DVB); Framing structure, channel coding and modulation for digital terrestrial television, July 1999, EN 300744 V1.2.1.

[4] The ESR5 criterion for the Assessment of DVB-T transmission Quality, ITU-R Document 6E/64-E, April 8, 2004.

[5] COST 207, "Digital land mobile radiocommunications," Office for Official Publications of the European Communities. Abschlussbericht, L\&emburg, 1989.

[6] SnapTrack, "Location Technologies for GSM, GPRS and UMTS Networks," Qualcomm Company, http://www.cdmatech.com/download_library/pdf/location_tec h_wp_1-03.pdf, 20.05.2007. 